\title{
Physiologically Based Pharmacokinetic Modeling for Substitutability Analysis of Venlafaxine Hydrochloride Extended-Release Formulations Using Different Release Mechanisms: Osmotic Pump versus Openable Matrix
}

\author{
Ho-pi Lin $^{l}$, Dajun Sun ${ }^{1}$, Xinyuan Zhang ${ }^{1,2}$, Hong Wen ${ }^{1,2}$
}

1. Office of Research and Standards, Office of Generic Drugs, Center for Drug Evaluation and Research, U.S. Food and Drug Administration, 10903 New Hampshire Avenue, Silver Spring, MD 20993, USA.

2. To whom correspondence should be addressed: (Xinyuan Zhang Tel: +1-240-402-7971; email: Xinyuan.Zhang@fda.hhs.gov, Hong Wen Tel: +1-240-402-7970, email: $\underline{\text { Hong.Wen@fda.hhs.gov). }}$ 


\begin{abstract}
An FDA-approved generic oral product of venlafaxine $\mathrm{HCl}$ ER tablets has employed a release mechanism based on an openable matrix, which is different from the push-pull osmotic pump system of its reference listed drug. In an extreme case, a delay in the bursting of the openable matrix may be considered a product failure mode that alters the intended profile of systemic exposure. A PBPK absorption model was established and verified to simulate the PK profiles after a single-dose oral administration of ER venlafaxine $\mathrm{HCl}$ tablets based on an osmotic pump or openable matrix design. This model adequately predicted the observed mean PK metrics human with less than $20 \%$ difference between the predicted and observed data. Based on the modeling and simulation results, Cmax and $\mathrm{AUC}_{\mathrm{t}}$ of the venlafaxine openable matrix tablets were entirely within the bioequivalence acceptance limits (i.e. $80 \%$ to $125 \%$ ) when the lag time varied from 0 to $4 \mathrm{hrs}$ and using drug release profiles under most dissolution conditions. The results indicated that a bioinequivalence risk is minimal for a delayed onset of drug release from the approved generic venlafaxine $\mathrm{HCl}$ ER tablets with an openable matrix design, supporting its substitutability to the reference product.
\end{abstract}

Keywords: Physiologically based pharmacokinetic modelling; osmotic pumps; controlled release; oral drug delivery; bioequivalence 


\section{Introduction}

The mechanisms used to achieve a controlled release in oral drug delivery systems are based on diverse and complex principles such as dissolution, diffusion, osmosis, swelling, and erosion. The development of push-pull osmotic pump systems and diffusion- or dissolution-controlled monolithic devices or reservoir systems has been widely applied to many extended-release (ER) oral dosage forms ${ }^{1}$. For the matrix-based ER formulations of Biopharmaceutics Classification System (BCS) Class I (i.e. high solubility, high permeability) drugs, common technical challenges include reducing the initial burst release, minimizing dose dumping, and achieving a constant release profile ${ }^{2,3}$. To overcome the issue of initial burst release ${ }^{3-7}$, one promising approach is to delay the onset of drug release by employing an openable layer in a formulation design known as a "wrap matrix" or "openable matrix", which can be found in several generic oral ER drug products such as venlafaxine hydrochloride ( $\mathrm{HCl})$ and levetiracetam ${ }^{9}$.

As illustrated in Figures 1A and 1B, the drug release from an osmotic pump is driven by the gradual swelling of a push layer upon ingress of the aqueous medium, whereas the swelling of an openable layer bursts open the wrap matrix, exposing the drug-release surface to the dissolution medium. For an openable matrix design, the core bi-layer tablet is coated with functional porous materials, using a mixture of insoluble (e.g. ethylcellulose) and soluble pore-forming excipients. In contrast to an osmotic pump design which has been thoroughly reviewed ${ }^{10,11}$, there have been relatively few investigations on the formulation-associated risk of an openable matrix which contains multiple compartments coated by a semi-permeable membrane.

Although ER tablets using the wrap matrix technology may accommodate various design modifications for different pharmaceutical requirements (Figure 1D), the drug release is governed by the same principle in which the swelling of the openable layer opens the matrix to expose the surface of the 
drug layer, facilitating a zero-order release due to a constant surface area. It is noteworthy that the time required to open the tablet counteracts the potential burst effect as commonly encountered in matrixbased ER tablets of freely water-soluble drugs as demonstrated in the hypothetical dissolution curves of Figure 1C. In this case, the onset of matrix breakage (i.e. a lag time of drug release) depends on the composition and thickness of functional coating of the openable layer and other factors such as pharmaceutical quality of coating film and a potential food effect. For instance, controlling a precise coating thickness of an openable matrix often poses technical challenges during the manufacturing process. Moreover, the effects of the openable layer composition and the in vivo micro-environment in the GI tract on the resulting breakage time of openable matrix tablets are not well understood.

At times, the generic products are designed using different release mechanisms from the reference listed drug (RLD) products. For example, a generic version of venlafaxine $\mathrm{HCl}$ ER tablets manufactured by Sun Pharmaceutical Industries Ltd. (Sun) adopted the "openable matrix" design ${ }^{8}$ while the RLD is designed as an osmotic pump ${ }^{12}$. Venlafaxine is a serotonin and norepinephrine reuptake inhibitor which has been formulated as IR and ER dosage forms for the treatment of depression among other neurological disorders ${ }^{13}$. Considering that the manufacturing variability of functional coating may affect the lag time of drug release from an openable matrix, setting suitable quality specifications to ensure consistent therapeutic performance is often under dispute. Hence, there is an imminent need of assessing the associated risk of the ER drug products using an openable matrix in order to establish clinically relevant specifications for quality control and to ensure consistent clinical performance.

Currently, there are two reference listed drug (RLD) products of oral ER venlafaxine hydrochloride based on diffusion-controlled coated beads encapsulated in capsules (Effexor XR by Wyeth Pharmaceuticals Inc. (now Pfizer), approved in 1997) and osmotic pump tablets (venlafaxine HCl ER tablets by Osmotica Pharmaceutical Corp., approved in 2008) on the U.S. market. In 2005, the 
Food and Drug Administration (FDA) approved a suitability petition submitted by Sun, requesting to develop a venlafaxine hydrochloride ER tablet dosage form using Effexor XR capsules as its RLD ${ }^{14}$. In this case, Sun adopted a formulation design using an openable matrix to achieve a sustained release of venlafaxine as previously described. After Sun has submitted its abbreviated new drug application (ANDA), Osmotica appealed that all pending ANDAs including Sun's application for venlafaxine hydrochloride ER tablets should cite Osmotica's drug product as the designated RLD via a citizen petition in $2008^{15}$. Given the regulatory, legal, and scientific considerations, the Agency determined that Sun's pending ANDA must be withdrawn and a new ANDA should be submitted to reference Osmotica's venlafaxine hydrochloride ER tablets ${ }^{16}$. Sun's generic venlafaxine hydrochloride ER tablets were eventually approved in August 2010, based on its demonstrated bioequivalence to Osmotica's product.

Physiologically based absorption modeling and simulation has been demonstrated to be powerful tools to assess in vivo performance and bioequivalence ${ }^{17}$ and has been applied in generic drug postmarketing surveillance and bioequivalence evaluation ${ }^{18-20}$. Using venlafaxine $\mathrm{HCl}$ as a model drug, the objective is to conduct physiologically based pharmacokinetic (PBPK) analysis to investigate the effect of different drug release profiles of an openable matrix on the resulting in vivo pharmacokinetic (PK) profiles compared with those of the RLD product using an osmotic pump. Based on the validated PBPK model for venlafaxine $\mathrm{HCl}$ ER products, simulations will be employed to evaluate the associated risks of various lag times and release profiles of an openable matrix design for oral ER dosage forms. The study results will not only confirm a minimal risk related to the lag-time effect of the generic version of venlafaxine $\mathrm{HCl}$ ER tablets based on an openable matrix, but also shed new lights on the risk assessment for this category of ER drug products. 


\section{Methods}

\subsection{Modeling and simulation strategy}

The development of the PBPK models, which simulate the plasma concentrations of venlafaxine and its major active metabolite, o-desmethylvenlafaxine (ODV), is based on the previously described methodology ${ }^{18,19}$. As illustrated in Figure 2, the modeling and simulation strategy begins with fitting compartmental models to the plasma concentration vs. time data following an IV administration of both venlafaxine and ODV to obtain the parameters describing the in vivo distribution, metabolism, and elimination kinetic processes. Next, the absorption models describing immediate-release (IR) venlafaxine $\mathrm{HCl}$ capsules (Effexor) were developed. Finally, the full physiologically based absorption models were established to predict the plasma concentrations of ER venlafaxine $\mathrm{HCl}$ capsules based on coated beads, tablets based on osmotic pumps, and tablets based on openable matrices.

\subsection{Human in vivo pharmacokinetic data}

Unless otherwise specified, all input parameters, dissolution profiles, and PK information were collected from multiple New Drug Applications (NDAs) and Abbreviated New Drug Applications (ANDAs). The observed clinical PK data of venlafaxine $\mathrm{HCl}$ ER capsules at $75 \mathrm{mg}$ and osmotic pump ER tablets at 75 mg can be found in the clinical pharmacology biopharmaceutics reviews of the new drug application ${ }^{21}$. The individual $\mathrm{PK}$ data points for IV venlafaxine, venlafaxine $\mathrm{HCl}$ osmotic pump tablets, and venlafaxine $\mathrm{HCl}$ openable matrix tablets are not available to the public.

\subsection{Model drug substances}

Venlafaxine is freely soluble in water with a measured aqueous solubility of $534 \mathrm{mg} / \mathrm{mL}^{22}$ and is considered a BCS Class I drug ${ }^{23,24}$. The major active metabolite (i.e. ODV), which is also freely soluble 
in water ${ }^{25}$, can be formed via cytochrome P450 (CYP) enzymes of CYP 2D6, 2C9, 2C19, and 3A4 in which the metabolic clearance through 2D6 is predominant ${ }^{26-29}$. The values for Vmax and Km used as a parameter input were obtained by in vitro and in vivo extrapolation ${ }^{29}$. The scaling factors for liver Vmax were obtained through model optimization while the $\mathrm{Km}$ value was kept the same as obtained in liver microsomes. All the physiochemical properties are summarized in Table 1.

\subsection{Pharmacokinetic models}

The PK data after an IV infusion of both venlafaxine and ODV were fitted in a one-, two-, or three- compartment model with linear and non-linear elimination in the PKPlus ${ }^{\mathrm{TM}}$ module of GastroPlus $^{\mathrm{TM}}$. The Hooke-and-Jeeves-Pattern-Search optimization and a unity weighting of the objective function were used to determine optimal compartmental model, clearance and distribution related parameters.

\subsection{Absorption/Advanced Compartmental Absorption and Transit (ACAT) models}

All the physiological parameters except those described below are model default values ${ }^{18}$. The conventional PK model from PKPlus ${ }^{\mathrm{TM}}$ module was re-optimized in the context of the Advanced Compartmental Absorption and Transit (ACAT) model built in GastroPlus ${ }^{\mathrm{TM}}$ (version 8.600034 , Simulations Plus Inc. Lancaster, CA) in which the clearance parameters from PKPlus ${ }^{\mathrm{TM}}$ were replaced by Vmax and $\mathrm{Km}$ values for CYP 2D6, 2C9, 2C19, and 3A4. In addition, the effective human permeability $\left(\mathrm{P}_{\text {eff }}\right)$ was obtained by fitting the model against the PK data of IR venlafaxine $\mathrm{HCl}$ tablets. The "Opt log D Model SA/V6.1" option was selected for determining the absorption scale factor.

The inputted PK parameters were fixed throughout all simulation trials, except for clearance of venlafaxine because the values are estimated based on trials conducted in different populations. Renal 
clearance was fixed in all models, considering its minimal role in drug elimination. The major route of clearance of venlafaxine was modeled by the gut and liver metabolism in which the individual values Vmax and $\mathrm{Km}$ for CYP 2D6, 2C9, 2C19, and 3A4 reported by Fogelman et al ${ }^{29}$ were used to establish the ACAT model for IV and oral dosage forms. Vmax values in liver were optimized by adjusting Vmax scaling factor per study population. In addition, the default values of enzyme distributions and expression levels were used in GastroPlus ${ }^{\mathrm{TM}}$.

Moreover, the dosage forms "IR: Tablet", "CR: Dispersed", and "Integral Tablet" were selected for immediate-release tablets, extended-release capsules, and extended-release tablets (including osmotic pumps and openable matrices), respectively, in GastroPlus ${ }^{\mathrm{TM}}$. A Weibull function describing the observed in vitro dissolution profiles for each ER formulation was used as the input function of in vitro dissolution during the simulation. Various dissolution profiles were tested in the PBPK models in order to select an optimal dissolution profile that best predicts the PK data of ER dosage forms. The dissolution profile which gives the highest value of $\mathrm{R}^{2}$ (coefficient of determination) was chosen to as the baseline value to predict the PK profiles, considering that $\mathrm{R}^{2}$ indicates how well the simulation predicts the observed data. Since venlafaxine oral dosage forms should be administered with food according to the drug label ${ }^{13}$, the observed PK profiles used for modeling and simulation here were collected from PK studies in healthy volunteers under fed conditions. Hence, a customized human fed physiology was optimized in GastroPlus ${ }^{\mathrm{TM}}$ in which absorption scaling factors (ASFs) for caecum and ascending colon were set to be the same as default values of physiological parameters in the ilium. As such, this allows for an appropriate description of plasma concentrations to reflect the late-stage absorption from caecum and colon.

\subsection{Parameter sensitivity analysis (PSA)}


To assess the impact of critical and uncertain parameters on in vivo performance, PSA was performed by varying one parameter at a time while keeping all other model parameters constant at the same time. PK metrics were calculated from the simulated plasma concentration-time profiles. In this case, PSA results were evaluated by comparing the value of each PK metric $\left(\mathrm{Cmax}\right.$ or $\left.\mathrm{AUC}_{\mathrm{t}}\right)$ associated with a parameter value to that of the corresponding metric in the baseline simulation. In addition, a Three-dimensional (3-D) PSA was conducted to assess the effect of tablet-opening time (i.e. a lag time of drug release) with respect to the sensitivity of a range of other input parameters. In such a 3-D PSA, two parameters including the lag time (e.g. 0, 1, 2, 3, $4 \mathrm{hrs)}$ and the parameters of interest (i.e. Vc, $\mathrm{k}_{12}$, $\mathrm{k}_{21}, \mathrm{k}_{13}, \mathrm{k}_{31}, \mathrm{Vmax}$ (gut), Km (gut), Vmax (liver), Km (liver), plasma unbound, blood plasma ratio, hepatic flow, stomach empty time, $\mathrm{P}_{\text {eff }}$, and drug solubility) were simultaneously tested for their impact on the resulting PK metrics $\left(\mathrm{Cmax}\right.$ and $\mathrm{AUC}_{\mathrm{t}}$ ), while all other model parameters remained constant. This simulation tested the in vivo pharmacokinetics of an openable matrix tablet formulation (reflected in the lag time) in a wide range of populations (reflected in the physiological and PK parameters). 


\section{Results}

3.1 Modeling and simulation of PK profiles after single-dose oral administration of IR and ER venlafaxine $\mathrm{HCl}$ formulations

Following the modeling and simulation steps illustrated in Figure 2, the observed and simulated PK profiles for drug products containing venlafaxine or o-desmethylvenlafaxine (ODV) are shown in Figure 3. The mean observed IV data for plasma concentrations of venlafaxine (10 mg) and ODV (50 $\mathrm{mg})^{32}$ were separately fitted using PKPlus ${ }^{\mathrm{TM}}$ (Figures $3 \mathrm{~A}$ and $\mathrm{B}$ ). In this case, three- and twocompartmental models could adequately predict the mean PK profiles for venlafaxine and ODV, respectively. The simulated data for venlafaxine $\mathrm{HCl}$ IR tablets are not shown here.

Next, PK profiles were simulated for three oral ER dosage forms including ER capsules (Figure 3C), ER tablets based on an osmotic pump at 75 and $150 \mathrm{mg}$ (Figures 3D and E) and an openable matrix at $150 \mathrm{mg}$ (Figure 3F). Several in vitro dissolution profiles (Figure 4) under different dissolution conditions (Table 2) were tested for best fitting the observed PK profiles of venlafaxine ER tablets in clinical studies. In this case, profile No. 4 (Figure 4 and Table 2) using a two-stage dissolution method was eventually selected as the input in vitro dissolution data owing to an accurate prediction of observed clinical PK data as well as its physiological relevance. As can be seen in Figures 3D and E, the predicted PK profiles of venlafaxine $\mathrm{HCl}$ osmotic pump ER tablets at $150 \mathrm{mg}$ are fairly consistent with those of the observed PK data, using both the dissolution profile No. 4 and the metabolism and elimination kinetics based on the IV infusion data of venlafaxine and ODV.

Nevertheless, the predicted PK metrics (i.e. Cmax and $\mathrm{AUC}_{\mathrm{t}}$ ) of the parent drug (venlafaxine) are within $20 \%$ of the observed mean $\mathrm{Cmax}$ and $\mathrm{AUC}_{\mathrm{t}}$ for all simulation (Figure 3). In addition, the general trend of venlafaxine PK profiles agrees well between the experimental data and model prediction as shown in Figures 3B, C, and D. The PK parameters for the metabolite ODV (e.g. volume 
of distribution, clearance, $\mathrm{k}_{12}, \mathrm{k}_{21}$ ), estimated from the ODV IV infusion data, were kept constant without further optimization across all study populations. This may, in part, account for the underestimated Cmax and $\mathrm{AUC}_{\mathrm{t}}$ of ODV PK profiles as shown in Figures 3C and 3D. It is understood that the PK parameters describing the metabolism and elimination processes of ODV in a specific population may not be equivalent to those from a different population. Considering that the BE requirement criteria of venlafaxine $\mathrm{HCl}$ ER tablets is based on the parent drug, the underestimated $\mathrm{PK}$ metrics for ODV should not affect the current BE assessment.

\subsection{Effect of in vivo dissolution profile on bioequivalence}

As illustrated in Figure 4, the drug release profiles of venlafaxine $\mathrm{HCl}$ ER tablets based on an osmotic pump are similar under different dissolution conditions (No. $1-3$ solid lines). In contrast, the release profiles of venlafaxine $\mathrm{HCl}$ ER tablets based on an openable matrix (No. 4 - No. 8 dashed lines in Figure 4) show a greater variation under different dissolution conditions (e.g. various medium $\mathrm{pH}$ and composition) compared to that of osmotic pumps. The findings are consistent with the fact that drug release from a tablet formulation based on an osmotic pump design into the GI lumen is independent of pH or gastric motility ${ }^{10,11}$.

The dissolution profile of openable matrix venlafaxine $\mathrm{HCl}$ ER tablets using a two-stage dissolution method (acidic condition followed by the $\mathrm{pH} 6.8$ medium) was chosen as the baseline input dissolution in the established PBPK models as it can reasonably predict the observed PK profiles (Figure 3F). To evaluate the influence of various dissolution profiles on bioequivalence, the PK metrics of the resulting simulated concentration-time curves using dissolution profiles under different conditions (Figure 4) were compared with those using the dissolution profile using the two-stage method (No. 4). As shown in Table 2 , the simulated $\% \mathrm{Cmax}$ and $\% \mathrm{AUC}_{\mathrm{t}}$ using majority dissolution profiles fall within 
the bioequivalence acceptance limits of $80 \%$ (lower) and $125 \%$ (upper), except for \%Cmax of the PK profile based on dissolution profile No. $5(\mathrm{pH}$ 1.2). However, this should not be a concern as it is unrealistic for the tablet to be exposed to such a low $\mathrm{pH}$ in the entire GI tract. Furthermore, two dissolution profiles plotted in black dots in Figure 4 represent the limits of $80 \%$ (lower) and 125\% Cmax compared to that of Sun's venlafaxine $\mathrm{HCl}$ ER tablets using the baseline dissolution profile (No. 4). To this end, the dissolution variations of the venlafaxine $\mathrm{HCl}$ ER tablets based on an openable matrix formulation have a minimal risk of bioinequivalence based on the established PBPK absorption model.

\subsection{Effect of lag time on bioequivalence}

To evaluate the impact of lag time of an openable layer on resulting simulated in vivo PK and bioequivalence, dissolution profiles with various lag times from 0 (baseline) to $4 \mathrm{hrs}$ in a Weibull function (parameters derived from two stage dissolution profile with lag time set to zero) were generated as shown in Figure 5. After inputting the hypothetical dissolution profiles into the established PBPK models, the resulting virtual trial shows that the longer it takes the openable layer to burst open, the lower the Cmax and $\mathrm{AUC}_{\mathrm{t}}$ due to a delayed onset of in vivo drug release (see Figure 6). Nevertheless, when the lag time varies from 0 to 4 hrs, the simulated results show that the PK metrics (i.e. Cmax and $\mathrm{AUC}_{\mathrm{t}}$ ) are entirely within the bioequivalence acceptance limits of $80 \%$ to $125 \%$. In this regard, the impact of the opening time of openable matrix venlafaxine $\mathrm{HCl}$ ER tablets on bioequivalence should have a minimal risk. Moreover, 3 -D sensitivity analyses using a lag time of $0,1,2,3$, and $4 \mathrm{hrs}$ as the $1^{\text {st }}$ dimension and various input parameters as the $2^{\text {nd }}$ dimension were conducted to review the risks associated with the lag time in simulated PK profiles (Table 3). The predictions show no dramatic change of $\mathrm{Cmax}$ and $\mathrm{AUC}_{\mathrm{t}}$ within the range tested, indicating that the lag time of drug release for an 
openable matrix is not considered a crucial factor affecting the resulting simulated bioequivalence for venlafaxine $\mathrm{HCl}$ ER tablets. 


\section{Discussion}

Physiologically based pharmacokinetic (PBPK) modeling has been utilized to assess the food effect $^{33,34}$, drug-drug interaction ${ }^{35-37}$, inter-batch quality variation ${ }^{38}$, in vivo performance in subpopulation $^{18}$, and bioequivalence for generic drug evaluation ${ }^{18,38,39}$. In the current study, we extended such a PBPK approach to assess the in vivo PK and bioequivalence associated with formulation release mechanism change for oral ER venlafaxine $\mathrm{HCl}$ dosage forms based on an openable matrix design. The development of an absorption model for venlafaxine was built in GastroPlus ${ }^{\mathrm{TM}}$ to predict the PK and bioequivalence outcome of matrix-based ER tablets. As illustrated in Figure 3, the predicted PK profiles of venlafaxine were reasonably consistent with the observed data after IV infusion of venlafaxine (10 $\mathrm{mg}$ ) and a single oral administration of ER capsules ( $75 \mathrm{mg}$ ) and ER tablets based on both osmotic pump (75 and $150 \mathrm{mg}$ ) and openable matrix $(150 \mathrm{mg}$ ). Based on the model validation against the human PK data, the established absorption model was deem sufficiently robust for the prediction of Cmax (predicted/observed value, 98\% - 116.2\%) and $\mathrm{AUC}_{\mathrm{t}}$ (predicted/observed value, $80.3 \%-95.8 \%$ ) for all the ER dosage forms. It is noteworthy that the systemic exposure was slightly underestimated probably due to the incomplete drug release as indicated by the in vitro dissolution profiles (Figure 4).

The current product-specific guidance on venlafaxine $\mathrm{HCl}$ ER oral dosage forms recommends ANDA applicants to conduct a 2-way crossover bioequivalence study at equivalent $150 \mathrm{mg}$ base in healthy volunteers under fed conditions due to a safety concern of administration of venlafaxine under fasting condition ${ }^{40,41}$. The simulation conditions specified in the Methods section are consistent with the current bioequivalence recommendations. In this case, Office of Generic Drugs recommends that both the parent drug (venlafaxine) and its metabolite (ODV) to be measured in plasma. The bioequivalence is recommended to be demonstrated based on the parent drug per the guidance. The similarity between the predicted and observed Cmax and $\mathrm{AUC}_{\mathrm{t}}$ for both venlafaxine and ODV (Figures 3B, C and D) shows 
that the established physiologically based absorption models can properly simulate the observed clinical PK data of venlafaxine $\mathrm{HCl}$ ER oral drug products.

Subsequently, several PBPK simulations were conducted to critically assess the potential risks of varying in vitro dissolution profiles and lag times of drug release for the venlafaxine $\mathrm{HCl}$ ER tablets using an openable matrix design. In contrast to drug release from osmotic pump systems ${ }^{11}$, factors such as gastric $\mathrm{pH}$, food effect, and other physiological parameters (e.g. gastric emptying rate) may influence drug release from conventional controlled-release systems (e.g. coated beads and matrix). Thus, it is not surprising that the dissolution profiles of the venlafaxine ER openable matrix (No. 4 - No. 8 in Figure 4) under different dissolution conditions show a higher variability in comparison to those of the venlafaxine ER osmotic pumps (No. 1 - No. 3 in Figure 4). When inputting these dissolution data into the established PBPK model, the predicted PK parameters after administrating a single dose of venlafaxine $\mathrm{HCl}$ ER tablets based on an openable matrix are shown to be bioequivalent to that using the two-stage dissolution method (i.e. the most physiologically relevant and accurately predicting the PK profiles), except for profile No. 5. In this case, venlafaxine initial release from an openable matrix tablet is slower in an acidic buffer (profile No. 5) than that of two-stage buffer (profile No. 4), resulting the simulated Cmax (64.3\%) falling outside of the bioequivalence requirement.

Moreover, PBPK simulations demonstrate that the lag time in which an openable matrix bursts open for the onset of venlafaxine release has a minimal effect on Cmax and a relatively small effect on $\mathrm{AUC}_{\mathrm{t}}$ (Figure 6) up to $4 \mathrm{hrs}$ of lag time. In this instance, a delayed onset of drug release for venlafaxine $\mathrm{HCl}$ ER tablets does not significantly influence the Cmax values for a single-dose bioequivalence study although the Tmax is clearly delayed. Considering that the plasma concentrations do not return to the baseline for more than $40 \mathrm{hrs}$ and the half-life is approximately $10.7 \mathrm{hrs}$ for venlafaxine $\mathrm{HCl} \mathrm{ER}$ tablets $^{13}$, PBPK simulations predict that the systemic exposure of an openable matrix with 4-hr lag time 
is approximately $80 \%$ of that with no lag time, conforming with the bioequivalence requirements for oral generic drug products. In addition, a minimal risk of bioinequivalence was confirmed in a 3-D sensitivity analysis using lag time (0-4 hrs) and various input parameters (Table 3$)$. To this end, the modeling and simulation results have verified the delayed opening and various drug release profiles do not significantly alter the resulting $\mathrm{PK}$ metrics $\left(\mathrm{Cmax}\right.$ and $\left.\mathrm{AUC}_{\mathrm{t}}\right)$ of the generic version of venlafaxine $\mathrm{HCl}$ ER tablets based on an openable matrix. We also noticed that the delayed onset of drug release will shift the PK profiles (Figure 6) suggesting that trough concentration at a steady state could be changed. However, in the absence of evidence suggesting that the trough venlafaxine concentration of the PK profile in an exposure-response relationship is associated with efficacy or safety in patients with panic disorder, a multiple-dose simulation was therefore not performed.

A question that arises with establishing a PBPK absorption model is related to choosing an adequate in vitro drug release profile as a model dissolution input for in vivo drug release. In the current study, it is expected that the in vitro release profile of venlafaxine $\mathrm{HCl}$ ER tablets can be used to sufficiently predict the in vivo $\mathrm{PK}$ profiles, considering that venlafaxine $\mathrm{HCl}$ is a highly soluble and highly permeable drug with a moderate half-life. In the case of osmotic pump ER tablets, the dissolution conditions (e.g. medium $\mathrm{pH})$ do not significantly affect the drug release during in vitro dissolution, leading to similar dissolution profiles under different conditions (Figure 4) generating a reasonable PK prediction (Figures 3D and E). In the case of openable matrix ER tablets, the selection of a bio-relevant in vitro dissolution profile as the model input is more complicated as drug release from an openable matrix is affected by a number of factors such as $\mathrm{pH}$ and residency time in each segment of the GI tract. For instance, Figure 4 shows that the dissolution rate of the openable matrix venlafaxine $\mathrm{HCl}$ ER tablets increases with an increasing $\mathrm{pH}$ of dissolution media. With this in mind, a dissolution condition based on the two-stage method $(\mathrm{pH} 1.5$ followed by $\mathrm{pH}$ 6.8) was eventually selected because it is considered 
the most in vivo relevant and predictive to model the PK profiles for openable matrix venlafaxine $\mathrm{HCl}$ ER tablets (Figure 3F). Given above scientific rationales, determining an appropriate dissolution profile for establishing PBPK absorption models should be based on the physicochemical properties of the drug substance and factors which may affect both in vitro and in vivo drug release of matrix-based tablet formulations. 


\section{Conclusion}

To summarize, we developed a PBPK absorption model for venlafaxine HCl ER formulations using the in vitro drug release profiles based on a two-stage method as the dissolution input to predict the PK profiles after a single-dose oral administration of venlafaxine $\mathrm{HCl}$ openable matrix and osmotic pump ER tablets. The established models were used to investigate a bioinequivalence risk associated with the potential delayed opening time of the openable matrix venlafaxine $\mathrm{HCl}$ ER tablets. Based on the single-dose PK simulations, a delayed onset of venlafaxine release up to $4 \mathrm{hrs}$ conforms to the bioequivalence requirements of $\mathrm{PK}$ metrics (i.e. $\mathrm{Cmax}$ and $\mathrm{AUC}_{\mathrm{t}}$ ), although the Tmax is expected to be delayed. Virtual clinical trials based on PBPK modeling and simulation can be a powerful tool to critically evaluate quality-associated risks on resulting formulation, drug release, pharmacokinetics and bioequivalence for generic drug products. 


\section{Acknowledgements}

The authors would like acknowledge scientific discussions and support of Drs. Robert Lionberger, Wenlei Jiang, Liang Zhao, Sarah Dutcher, and Ming Qin. This was supported in part by an appointment to the ORISE Research Participation Program at the Center for Drug Evaluation and Research, administered by the Oak Ridge Institute for Science and Education through an interagency agreement between the US Department of Energy and the US Food and Drug Administration.

\section{Disclaimer}

This article reflects the views of the authors and should not be construed to represent the FDA's views or policies. 


\section{References}

1. Wen H, Park K. 2010. Introduction and an overview of oral controlled release formulation design. New York: John Wiley.

2. Huang X, Brazel CS 2001. On the importance and mechanisms of burst release in matrixcontrolled drug delivery systems. J Control Release 73(2):121-136.

3. Zeng W 2004. Oral controlled release formulation for highly water-soluble drugs: drug-sodium alginate-xanthan gum-zinc acetate matrix. Drug Dev Ind Pharm 30(5):491-495.

4. Gohel MC, Bariya SH 2009. Fabrication of triple-layer matrix tablets of venlafaxine hydrochloride using xanthan gum. AAPS PharmSciTech 10(2):624-630.

5. Aboelwafa AA, Basalious EB 2010. Optimization and in vivo pharmacokinetic study of a novel controlled release venlafaxine hydrochloride three-layer tablet. AAPS PharmSciTech 11(3):1026-1037.

6. Peng Y, Li J, Li J, Fei Y, Dong J, Pan W 2013. Optimization of thermosensitive chitosan hydrogels for the sustained delivery of venlafaxine hydrochloride. Int J Pharm 441(1):482-490.

7. Tian L, Zhang Y, Tang X 2008. Sustained-release pellets prepared by combination of wax matrices and double-layer coatings for extremely water-soluble drugs. Drug Dev Ind Pharm 34(6):569576.

8. Zala YR, Dharmadhikari NB. 2006. Pharmaceutical Composition. Sun Pharma Advanced Research Company Ltd. assignee. Patent US 8431156 B2. February 22, 2006.

9. Zala YR, Dharmadhikari NB. 2011. Pharmaceutical Composition. Sun Pharma Advanced Research Company Ltd. assignee. Patent US8425938 B2. May 06, 2011.

10. Santus G, Baker RW 1995. Osmotic drug delivery: a review of the patent literature. J Control Release 35(1):1-21. 
11. Verma RK, Krishna DM, Garg S 2002. Formulation aspects in the development of osmotically controlled oral drug delivery systems. J Control Release 79(1):7-27.

12. Meyer GA, Ricci MA, Bigatti G. 2006. Venlafaxine osmotic device formulation. Assignee: Meyer GA, Ricci MA, Bigatti G. assignee. Patent US20070077301 A.1. September 15, 2006.

13. 1993. Osmotica, Venlafaxine Extended Release Tablets (venlafaxine hydrochloride) [package insert].

14. Buehler GJ. 2005. Citizen petition: Requiring permission to file an Abbreviated New Drug Application (ANDA) for the following drug products: Venlafaxine Hydrochloride Extended-release Tablets, Docket No. FDA-2003P-0159/CP1. The U.S. Department of Health and Human Services (HHS), Food and Drug Administration (FDA).

15. Aikman MS. 2008. Citizen petition: Requiring the submission of a new ANDA containing the required bioequivalence data for venlafaxine $\mathrm{HCl}$ extended-release tablets, Docket No. FDA-2008-P0329-0001. The U.S. Department of Health and Human Services (HHS), Food and Drug Administration (FDA).

16. Woodcock J. 2008. Response to citizen petition: Requiring the submission of a new ANDA containing the required bioequivalence data for venlafaxine $\mathrm{HCl}$ extended-release tablets, Docket No. FDA-2008-P-0329-0016. The U.S. Department of Health and Human Services (HHS), Food and Drug Administration (FDA)

17. Kostewicz ES, Aarons L, Bergstrand M, Bolger MB, Galetin A, Hatley O, Jamei M, Lloyd R, Pepin X, Rostami-Hodjegan A 2014. PBPK models for the prediction of in vivo performance of oral dosage forms. Eur J Pharm Sci 57:300-321.

18. Babiskin AH, Zhang X 2015. Application of Physiologically Based Absorption Modeling for Amphetamine Salts Drug Products in Generic Drug Evaluation. J Pharm Sci 104(9):3170-3182. 
19. Zhang X, Lionberger RA, Davit BM, Yu LX 2011. Utility of physiologically based absorption modeling in implementing Quality by Design in drug development. AAPS J 13(1):59-71.

20. Zhang X, Lionberger R 2014. Modeling and simulation of biopharmaceutical performance. Clin Pharmacol Ther 95(5):480-482.

21. 2008. NDA 22-104 Clinical pharmacology and biopharmaceutics review(s), Drugs@FDA (Link http://www.accessdata.fda.gov/drugsatfda_docs/nda/2008/022104s000_ClinPharmR.pdf). The U.S. Department of Health and Human Services (HHS), Food and Drug Administration (FDA).

22. Patel HA, Shah S, Shah DO, Joshi P 2011. Sustained release of venlafaxine from venlafaxinemontmorillonite-polyvinylpyrrolidone composites. Appl Clay Sci 51(1):126-130.

23. 2010. Draft guidance on venlafaxine hydrochloride (immediate release). The U.S. Department of Health and Human Services (HHS), Food and Drug Administration (FDA).

24. Kasim NA, Whitehouse M, Ramachandran C, Bermejo M, Lennernäs H, Hussain AS, Junginger HE, Stavchansky SA, Midha KK, Shah VP 2004. Molecular properties of WHO essential drugs and provisional biopharmaceutical classification. Mol Pharmaceut 1(1):85-96.

25. Vandenberg AM 2014. What is the difference? Within class comparisons of psychotropic agents with new products. Ment Health Clin 4(1):2-7.

26. Goeringer KE, McIntyre IM, Drummer OH 2001. Postmortem tissue concentrations of venlafaxine. Forensic Sci Int 121(1):70-75.

27. Shams M, Arneth B, Hiemke C, Dragicevic A, Müller M, Kaiser R, Lackner K, Härtter S 2006. CYP2D6 polymorphism and clinical effect of the antidepressant venlafaxine. J Clin Pharm Ther 31(5):493-502.

28. Ereshefsky L 1996. Drug-drug interactions involving antidepressants: focus on venlafaxine. $J$ Clin Psychopharm 16(3):37S-50S. 
29. Fogelman SM, Schmider J, Venkatakrishnan K, von Moltke LL, Harmatz JS, Shader RI, Greenblatt DJ 1999. O-and N-demethylation of venlafaxine in vitro by human liver microsomes and by microsomes from cDNA-transfected cells: effect of metabolic inhibitors and SSRI antidepressants. Neuropsychopharmacology 20(5):480-490.

30. Siccardi M, Marzolini C, Seden K, Almond L, Kirov A, Khoo S, Owen A, Back D 2013. Prediction of drug-drug interactions between various antidepressants and efavirenz or boosted protease inhibitors using a physiologically based pharmacokinetic modelling approach. Clin Pharmacokinet 52(7):583-592.

31. Troy SM, Parker VP, Hicks DR, Pollack GM, Chiang ST 1997. Pharmacokinetics and effect of food on the bioavailability of orally administered venlafaxine. J Clin Pharm 37(10):954-961.

32. Nichols AI, Behrle JA, Richards LS, Parker VD, Posener JA, Fruncillo R, Paul J 2013. The absolute bioavailability of desvenlafaxine in healthy subjects. J Bioequiv Availab 4(2):18-23. 33. Xia B, Heimbach T, Lin T-h, Li S, Zhang H, Sheng J, He H 2013. Utility of physiologically based modeling and preclinical in vitro/in vivo data to mitigate positive food effect in a BCS class 2 compound. AAPS PharmSciTech 14(3):1255-1266.

34. Parrott N, Lukacova V, Fraczkiewicz G, Bolger M 2009. Predicting pharmacokinetics of drugs using physiologically based modeling—application to food effects. AAPS J 11(1):45-53.

35. Varma MV, Lai Y, Feng B, Litchfield J, Goosen TC, Bergman A 2012. Physiologically based modeling of pravastatin transporter-mediated hepatobiliary disposition and drug-drug interactions. Pharm Res 29(10):2860-2873.

36. Vieira ML, Zhao P, Berglund E, Reynolds K, Zhang L, Lesko L, Huang SM 2012. Predicting Drug Interaction Potential With a Physiologically Based Pharmacokinetic Model: A Case Study of Telithromycin, a Time-Dependent CYP3A Inhibitor. Clin Pharm Ther 91(4):700-708. 
37. Wagner C, Thelen K, Willmann S, Selen A, Dressman JB 2013. Utilizing in vitro and PBPK tools to link ADME characteristics to plasma profiles: case example nifedipine immediate release formulation. J Pharm Sci 102(9):3205-3219.

38. Mitra A, Kesisoglou F, Dogterom P 2015. Application of absorption modeling to predict bioequivalence outcome of two batches of etoricoxib tablets. AAPS PharmSciTech 16(1):76-84. 39. Cristofoletti R, Dressman JB 2014. Use of physiologically based pharmacokinetic models coupled with pharmacodynamic models to assess the clinical relevance of current bioequivalence criteria for generic drug products containing ibuprofen. J Pharm Sci 103(10):3263-3275.

40. 2008. Draft guidance on venlafaxine hydrochloride (extended release capsules/oral). The U.S. Department of Health and Human Services (HHS), Food and Drug Administration (FDA).

41. 2010. Draft guidance on venlafaxine hydrochloride (extended release tablets/oral). The U.S. Department of Health and Human Services (HHS), Food and Drug Administration (FDA). 


\section{Figure/Table Legends}

Figure 1. Schematic illustration of drug release mechanisms from the formulations based on (A) an osmotic pump and (B) an openable matrix; (C) their corresponding kinetics of drug release; and (D) different formulation designs of the openable matrix drug delivery.

Figure 2. Schematic illustration of modeling and simulation strategy. The boxes of the same color represent the studies conducted in the same clinical trial.

Figure 3. Observed (refer to Section 2.1 for PK data collection) and predicted PK profiles for drug products containing venlafaxine or ODV for (A) venlafaxine IV infusion $(0.5 \mathrm{hr})$ at $10 \mathrm{mg}$, and (B) ODV IV infusion (1 hr) at $50 \mathrm{mg}^{32}$, (C) venlafaxine $\mathrm{HCl}$ ER capsules at $75 \mathrm{mg}^{21}$, (D) venlafaxine $\mathrm{HCl}$ osmotic pump ER tablets at $75 \mathrm{mg}^{21}$, (E) venlafaxine $\mathrm{HCl}$ osmotic pump ER tablets at $150 \mathrm{mg}$, and (F) venlafaxine $\mathrm{HCl}$ openable matrix $\mathrm{ER}$ tablets at $150 \mathrm{mg}$. Red: venlafaxine; blue: ODV; solid lines: simulated plasma concentrations; circle symbols: mean observed plasma concentrations. Inset: The Cmax (in $\mathrm{ng} / \mathrm{mL}$ ) and AUCt (ng hr/mL) values of observed and predicted pharmacokinetic profiles for venlafaxine are presented in (A), (C), (D), (E) and (F), and for ODV in (B).

Figure 4. The reported mean in vitro dissolution profiles (No. 1 - 3 solid lines for osmotic pump and No. 4 - 8 dashed lines for openable matrix) using (1) $\mathrm{pH} 1.2$, (2) $\mathrm{pH} 4.5$, (3) water, (4) two-stage (pH

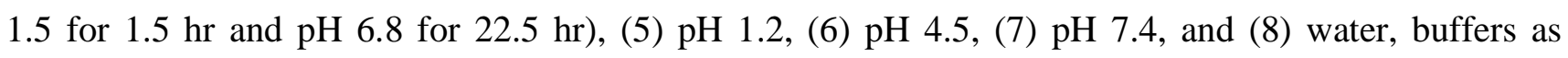
dissolution media in a USP II apparatus at an agitation rate of $50 \mathrm{rpm}$. By adjusting the parameters in a 
Weibull function of input dissolution profiles, two dissolution profiles plotted in black dots represent the lower and upper limits of $80 \%$ and $125 \%$ Cmax compared to that of Sun's venlafaxine hydrochloride ER tablets using dissolution profile No. 4.

Figure 5. Hypothetical dissolution profiles generated to test the effect of lag time on PK metrics (Cmax and $\mathrm{AUC}_{\mathrm{t}}$ ) through a Weilbull function with a lag time of 0 (baseline), 1, 2, 3, and $4 \mathrm{hr}$, while the rest of Weibull parameters were kept constant.

Figure 6. Effect of lag time $(0-4 \mathrm{hr})$ on simulated PK profiles based on the established PBPK models. Inset: Fractional $\mathrm{Cmax}$ and $\mathrm{AUC}_{\mathrm{t}}$ of each simulated $\mathrm{PK}$ profile compared to that with zero lag time.

Table 1. Summary of input parameters for venlafaxine and o-desmethylvenlafaxine (ODV) ACAT simulations

Table 2: Summary of $\% \mathrm{Cmax}$ and $\% \mathrm{AUC}_{\mathrm{t}}$ of simulated pharmacokinetic profiles using inputs of various in vitro dissolution profiles presented in Figure 3, compared to that using dissolution profile No. 4 for openable matrix venlafaxine $\mathrm{HCl}$ ER tablets.

Table 3: 3-D PSA using a lag time of $0,1,2,3,4 \mathrm{hr}\left(1^{\text {st }}\right.$ dimension $)$ against various input parameters (10 test conditions with a uniform spacing) $\left(2^{\text {nd }}\right.$ dimension) with total of 50 combinations per pair of parameters. For any particular test condition in $2^{\text {nd }}$ dimension, simulated Cmax or $\mathrm{AUC}_{\mathrm{t}}$ were compared 
to those generated from the conditions paired with a 0 -hr lag time. The range of $\% \mathrm{Cmax}$ and $\% \mathrm{AUC}_{\mathrm{t}}$ (minimum and maximum out of 40 results per pair of parameters) were reported in the table. 
Table 1. Summary of input parameters for venlafaxine and o-desmethylvenlafaxine (ODV) ACAT simulations

\begin{tabular}{|c|c|c|c|c|c|c|}
\hline Drug or Drug product & $\begin{array}{l}\text { ODV IV } \\
\text { Infusion }\end{array}$ & $\begin{array}{l}\text { Venlafaxine } \\
\text { IV Infusion }\end{array}$ & Effexor & Effexor XR & $\begin{array}{l}\text { ER tablet } \\
\text { (Osmotica) }\end{array}$ & $\begin{array}{l}\text { ER tablet } \\
\text { (Sun) }\end{array}$ \\
\hline Molecule & ODV & Venlafaxine & Venlafaxine & Venlafaxine & Venlafaxine & Venlafaxine \\
\hline Route of administration & IV infusion $1 \mathrm{~h}$ & $\begin{array}{c}\text { IV infusion } \\
0.5 \mathrm{~h}\end{array}$ & oral & oral & oral & oral \\
\hline Dose (mg) & 50 & 10 & 50 & 75 & 75 or 150 & 150 \\
\hline Dosage form ${ }^{a}$ & IV: Infusion & IV: Infusion & IR: Tablet & CR: Dispersed & $\begin{array}{l}\text { CR: Integral } \\
\text { Tablet }\end{array}$ & $\begin{array}{l}\text { CR: Integral } \\
\text { Tablet }\end{array}$ \\
\hline Dissolution model & N/A & N/A & N/A & Weibull & Weibull & Weibull \\
\hline Dose volume $(\mathrm{ml})$ & N/A & N/A & 240 & 240 & 240 & 240 \\
\hline Particle density $(\mathrm{g} / \mathrm{ml})^{\mathrm{a}}$ & N/A & N/A & 1.2 & 1.2 & 1.2 & 1.2 \\
\hline $\mathrm{MW}(\mathrm{g} / \mathrm{mol})$ & 263.38 & \multicolumn{5}{|c|}{277.41} \\
\hline $\log P$ & $2.62^{\mathrm{b}}$ & \multicolumn{5}{|c|}{$2.8^{28}$} \\
\hline Solubility (mg/ml) & $34.6(\mathrm{pH} 6.5)$ & \multicolumn{5}{|c|}{$572(\mathrm{pH} \mathrm{7})^{\mathbf{c}}$} \\
\hline $\mathrm{pKa}^{\mathrm{a}}$ & $\begin{array}{l}8.86 \text { (base) } \\
10.25 \text { (acid) }\end{array}$ & \multicolumn{5}{|c|}{8.94 (base) } \\
\hline $\begin{array}{l}\text { Diffusion coefficient } \\
\left(\mathrm{cm}^{2} / \mathrm{s} \times 10^{-5}\right)^{\mathrm{a}}\end{array}$ & 0.75 & \multicolumn{5}{|c|}{0.72} \\
\hline$f_{u p}(\%)^{d}$ & 70 & \multicolumn{5}{|c|}{73} \\
\hline $\mathbf{R}_{\mathrm{bp}}$ & $1.2^{\mathrm{b}}$ & \multicolumn{5}{|c|}{$1^{\mathrm{e}}$} \\
\hline Permeability $\left(\times 10^{-4} \mathrm{~cm} / \mathrm{s}\right)$ & $1.28^{\mathrm{b}}$ & \multicolumn{5}{|c|}{$1.668^{f}$} \\
\hline Central Volume $(\mathrm{L} / \mathrm{Kg})^{\mathrm{f}}$ & 1.2648 & \multicolumn{5}{|c|}{0.45939} \\
\hline$K_{12}(1 / h)^{f}$ & 3.1166 & \multicolumn{5}{|c|}{12.756} \\
\hline$K_{21}(1 / h)^{f}$ & 2.1747 & \multicolumn{5}{|c|}{2.6796} \\
\hline$K_{13}(1 / h)^{f}$ & 0 & \multicolumn{5}{|c|}{0.25792} \\
\hline$K_{31}(1 / h)^{f}$ & 0 & \multicolumn{5}{|c|}{0.04089} \\
\hline Clearance $(\mathrm{L} / \mathrm{h} / \mathrm{Kg})^{\mathrm{f}}$ & 0.25542 & \multicolumn{5}{|c|}{0} \\
\hline Renal clearance $(\mathrm{L} / \mathrm{h} / \mathrm{Kg})^{29}$ & 0 & \multicolumn{5}{|c|}{0.054} \\
\hline CYP2C19 O-demethylation ${ }^{27}$ & Vmax: 0; Km: 0 & \multicolumn{5}{|c|}{ Vmax: $0.17 \mathrm{mg} / \mathrm{s} / \mathrm{mg}$-enzyme; Km: $59.34 \mathrm{mg} / \mathrm{L}$} \\
\hline CYP2C19 N-demethylation ${ }^{27}$ & Vmax: 0; Km: 0 & \multicolumn{5}{|c|}{ Vmax: $0.33 \mathrm{mg} / \mathrm{s} / \mathrm{mg}$-enzyme; Km: $80.6 \mathrm{mg} / \mathrm{L}$} \\
\hline CYP2C9 O-demethylation ${ }^{27}$ & Vmax: 0; Km: 0 & \multicolumn{5}{|c|}{ Vmax: $0.13 \mathrm{mg} / \mathrm{s} / \mathrm{mg}$-enzyme; Km: $631.6 \mathrm{mg} / \mathrm{L}$} \\
\hline CYP2C9 N-demethylation ${ }^{27}$ & Vmax: 0; Km: 0 & \multicolumn{5}{|c|}{ Vmax: $2.38 \mathrm{mg} / \mathrm{s} / \mathrm{mg}$-enzyme; $\mathrm{Km}: 455.7 \mathrm{mg} / \mathrm{L}$} \\
\hline CYP2D6 O-demethylation ${ }^{27}$ & Vmax: 0; Km: 0 & \multicolumn{5}{|c|}{ Vmax: $0.16 \mathrm{mg} / \mathrm{s} / \mathrm{mg}$-enzyme; Km: $4.698 \mathrm{mg} / \mathrm{L}$} \\
\hline CYP3A4 N-demethylation ${ }^{27}$ & Vmax: 0; Km: 0 & \multicolumn{5}{|c|}{ Vmax: $0.43 \mathrm{mg} / \mathrm{s} / \mathrm{mg}$-enzyme; Km: $112.6 \mathrm{mg} / \mathrm{L}$} \\
\hline
\end{tabular}

\footnotetext{
${ }^{a}$ Dose form selected in GastroPlus

${ }^{\mathrm{b}}$ Predicted by ADME predictor or default values in GastroPlus

${ }^{\mathrm{c}}$ Reported in reviews from Drug@FDA

${ }^{\mathrm{d}}$ Fraction of the drug unbound reported in drug labels

e The blood-to-plasma partition coefficient ${ }^{29}$

${ }^{\mathrm{f}}$ Optimized values
} 
Table 2: Summary of $\% \mathrm{Cmax}$ and $\% \mathrm{AUC}_{\mathrm{t}}$ of simulated pharmacokinetic profiles using inputs of various in vitro dissolution profiles presented in Figure 3, compared to that using dissolution profile No. 4 for openable matrix venlafaxine $\mathrm{HCl}$ ER tablets.

\begin{tabular}{|c|c|c|c|c|}
\hline $\begin{array}{c}\text { Dissolution } \\
\text { profile }\end{array}$ & Dosage form & Dissolution medium & $\begin{array}{l}\text { \% Cmax compared to } \\
\text { two-stage dissolution }\end{array}$ & $\begin{array}{l}\% \mathrm{AUC}_{\mathrm{t}} \text { compared to } \\
\text { two-stage dissolution }\end{array}$ \\
\hline No. 1 & Osmotic pump & $\mathrm{pH} 1.2 \mathrm{HCl}$ & 101.1 & 92.2 \\
\hline No. 2 & Osmotic pump & pH 4.5 buffer & 104.0 & 92.7 \\
\hline No. 3 & Osmotic pump & Water & 111.0 & 97.3 \\
\hline No. 4 & Openable matrix & $\begin{array}{c}\text { two-stage (pH } 1.5 \text { for } 1.5 \\
\text { hr; } \mathrm{pH} 6.8 \text { for } 22.5 \mathrm{hr} \text { ) }\end{array}$ & 100.0 & 100.0 \\
\hline No. 5 & Openable matrix & $\mathrm{pH} 1.2 \mathrm{HCl}$ & 64.3 & 83.0 \\
\hline No. 6 & Openable matrix & $\mathrm{pH} 4.5$ buffer & 83.4 & 90.3 \\
\hline No. 7 & Openable matrix & pH 7.4 buffer & 97.5 & 99.6 \\
\hline No. 8 & Openable matrix & Water & 83.0 & 91.6 \\
\hline
\end{tabular}


Table 3: 3-D PSA using a lag time of $0,1,2,3,4 \mathrm{hr}\left(1^{\text {st }}\right.$ dimension) against various input parameters (10 test conditions with a uniform spacing) $\left(2^{\text {nd }}\right.$ dimension) with total of 50 combinations per pair of parameters. For any particular test condition in $2^{\text {nd }}$ dimension, simulated $\mathrm{Cmax}$ or $\mathrm{AUC}_{\mathrm{t}}$ were compared to those generated from the conditions paired with a 0 hr lag time. The range of $\% \mathrm{Cmax}$ and $\% \mathrm{AUC}_{\mathrm{t}}$ (minimum and maximum out of 40 results per pair of parameters) were reported in the table.

\begin{tabular}{|c|c|c|}
\hline \multirow{2}{*}{${\frac{1}{2^{\text {nd }}} \text { dimension }}^{\text {st }}$} & \multicolumn{2}{|c|}{$\begin{array}{l}\text { Lag time of } 1,2,3,4 \mathrm{hr} / \mathrm{lag} \\
\text { time } 0 \mathrm{hr}\end{array}$} \\
\hline & Cmax (\%) & $\mathrm{AUC}_{\mathrm{t}}(\%)$ \\
\hline $0.1-10 X \mathrm{Vc}$ & $93-100$ & $96-100$ \\
\hline $0.1-10 \mathrm{X} \mathrm{k}_{12}$ & $93-102$ & $97-100$ \\
\hline $0.1-10 \mathrm{X} \mathrm{k}_{21}$ & $93-100$ & $97-100$ \\
\hline $0.1-10 \mathrm{X} \mathrm{k}_{13}$ & $96-99$ & $97-100$ \\
\hline $0.1-10 \mathrm{X} \mathrm{k}_{31}$ & $97-99$ & $99-100$ \\
\hline $0.1-10 X$ Vmax (gut) & 94-101 & $95-103$ \\
\hline $1-10 X \mathrm{Km}$ (gut) & $95-105$ & $96-110$ \\
\hline 0.2-17X Vmax (liver) & $87-99$ & $95-99$ \\
\hline $0.1-10 \mathrm{X} \mathrm{Km}$ (liver) & $97-100$ & $97-100$ \\
\hline Plasma unbound (1-100\%) & $97-101$ & $96-100$ \\
\hline 0.5-10X Blood plasma ratio & $96-100$ & $98-100$ \\
\hline Hepatic flow $(0.25-2.5 \mathrm{X})$ & $96-99$ & $98-100$ \\
\hline Stomach empty time $(0.5-10 \mathrm{hr})$ & $86-112$ & $96-100$ \\
\hline $0.1-10 \mathrm{X} \quad \mathrm{P}_{\text {eff }}$ & $93-99$ & $95-100$ \\
\hline
\end{tabular}




\section{(A) Osmotic pump}

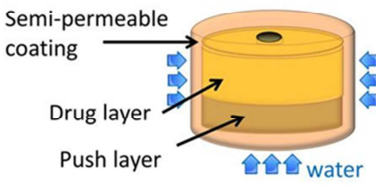

(B) Openable matrix

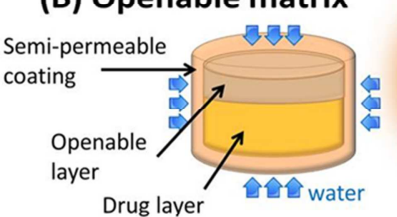

(C)
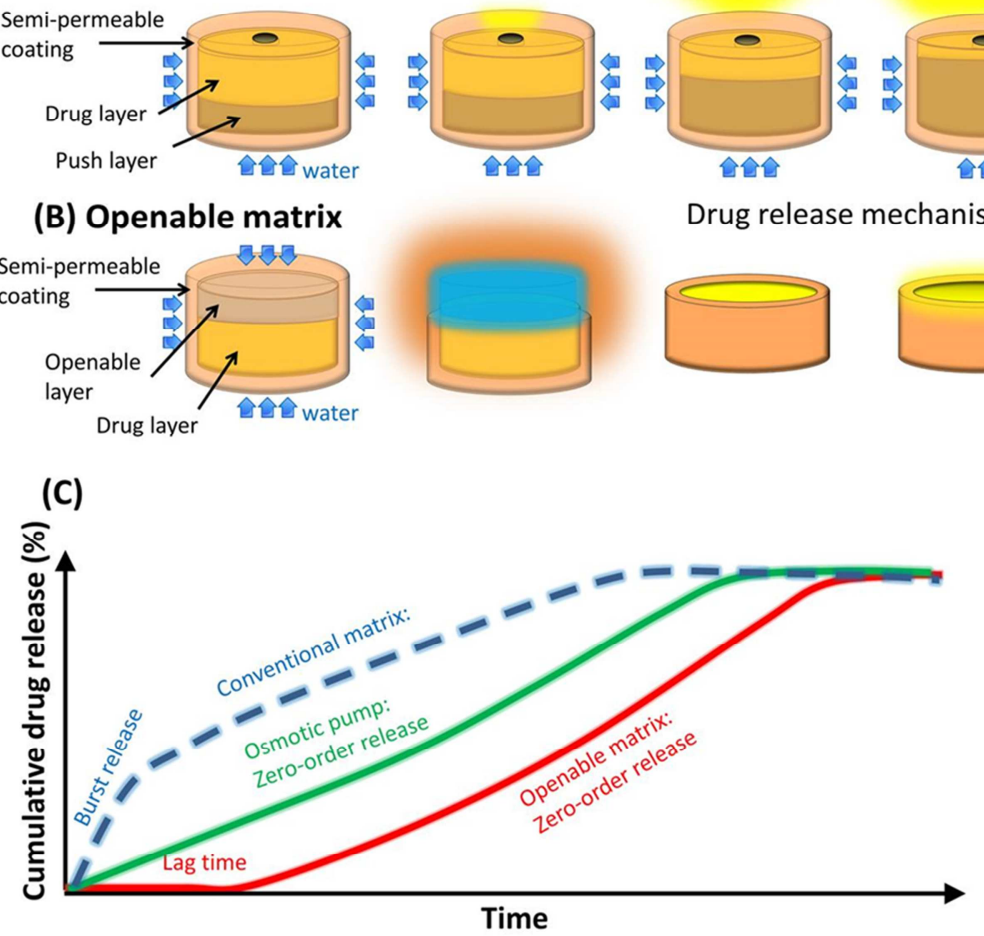

습습
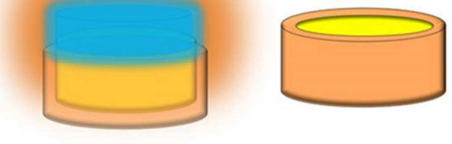
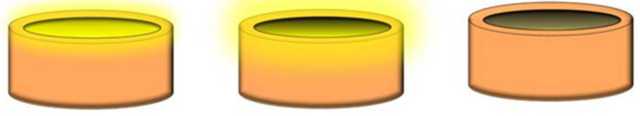

(D)

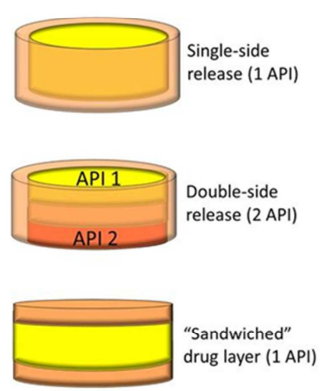




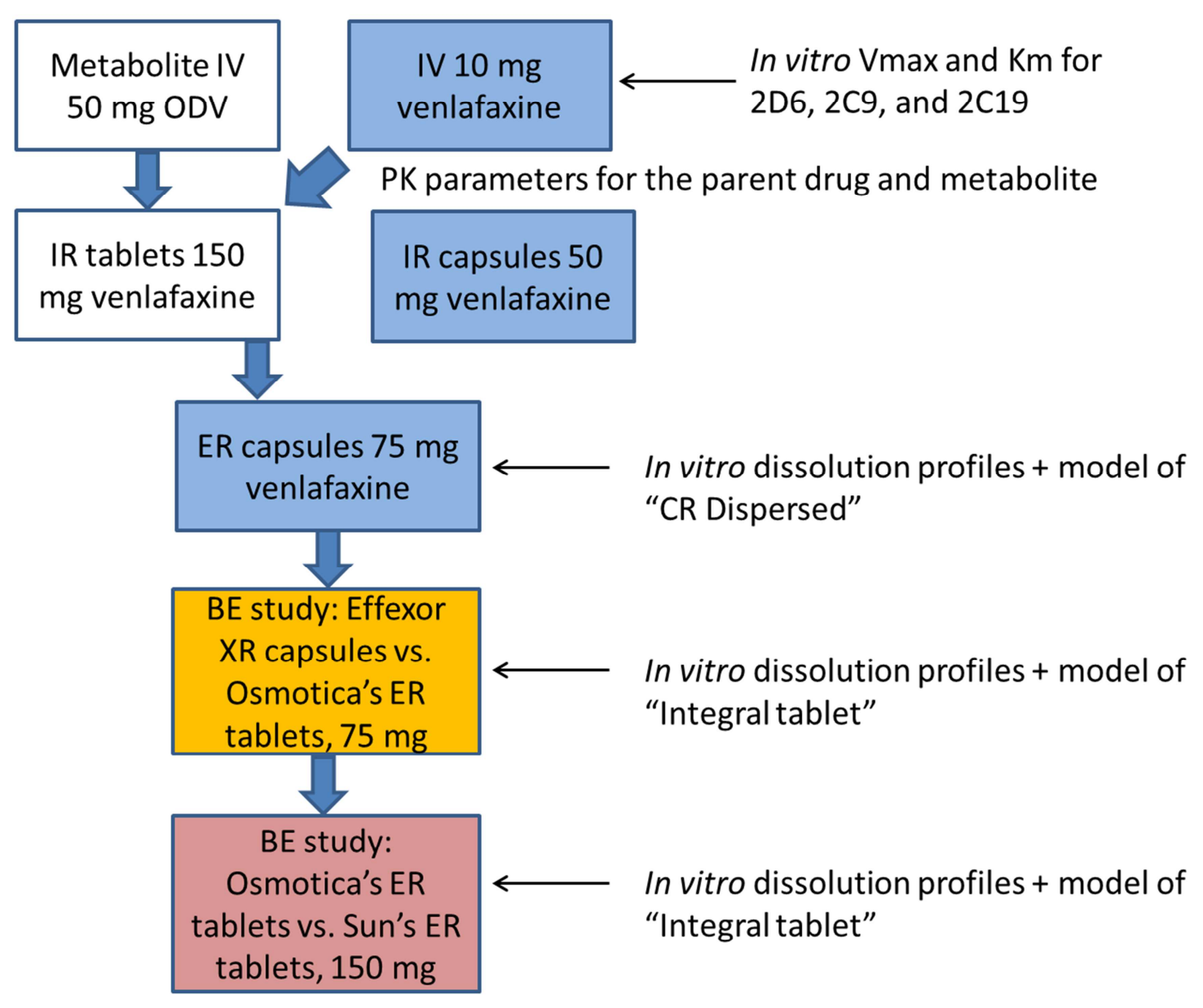



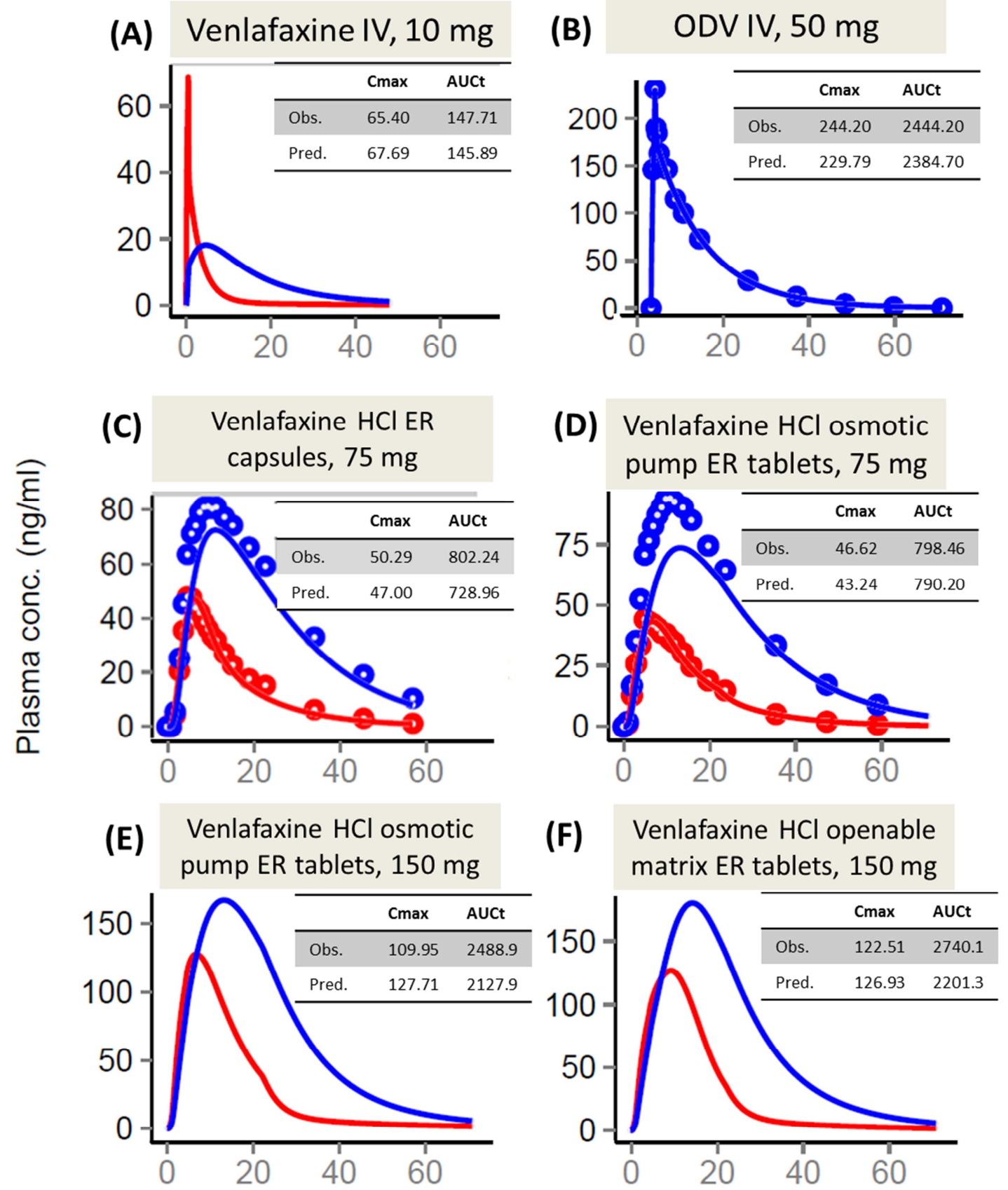

Time (hr) 


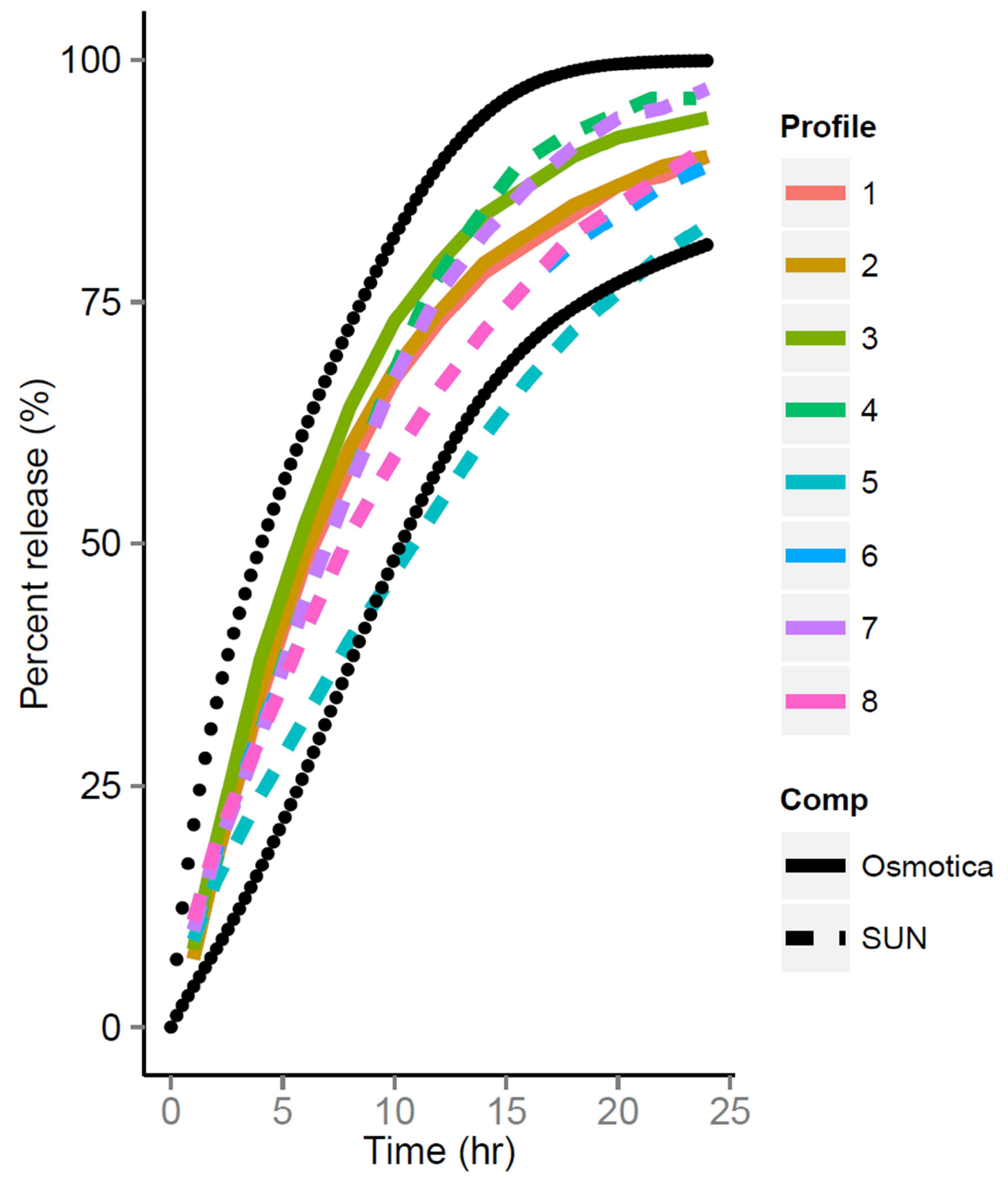




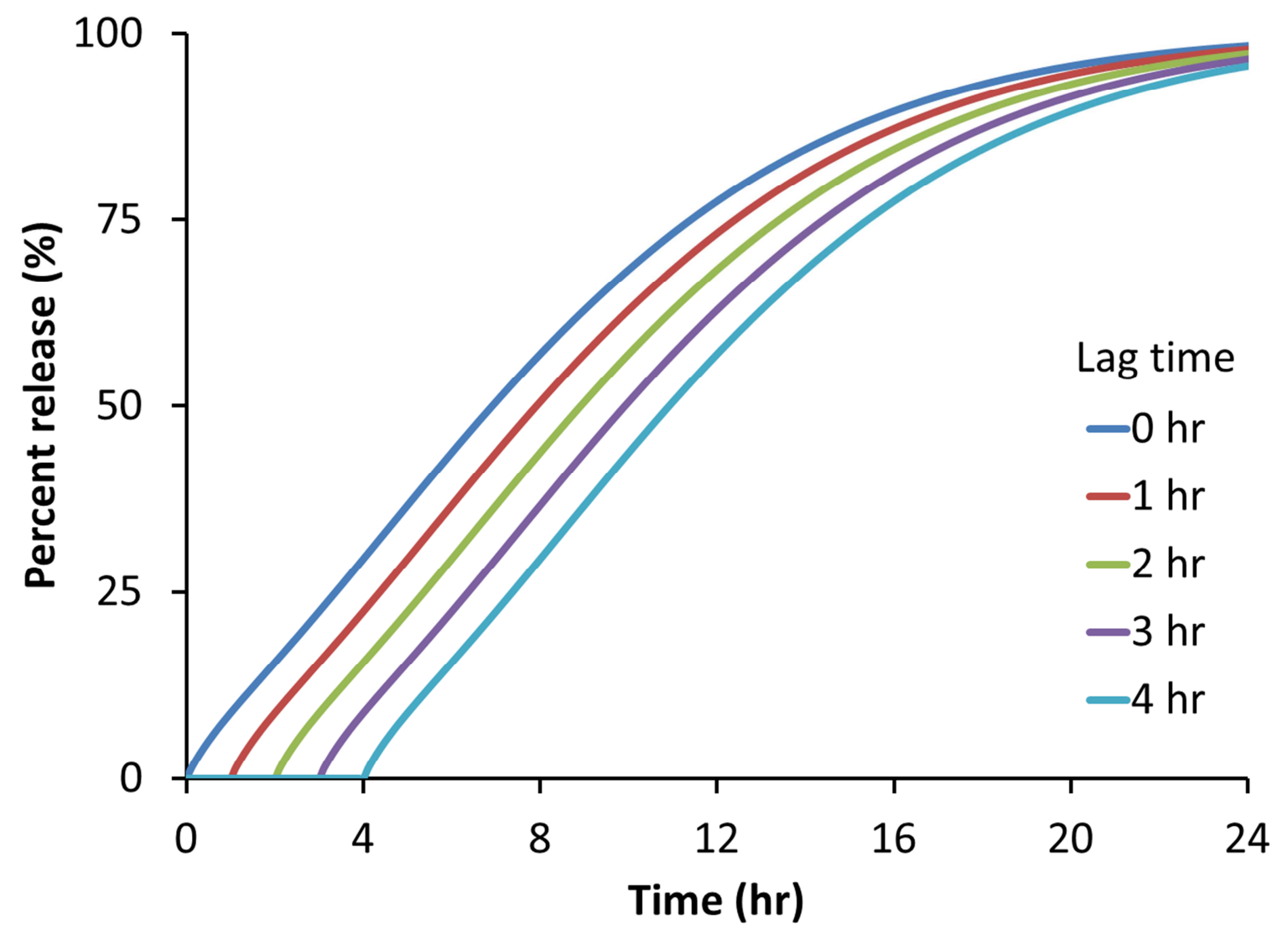




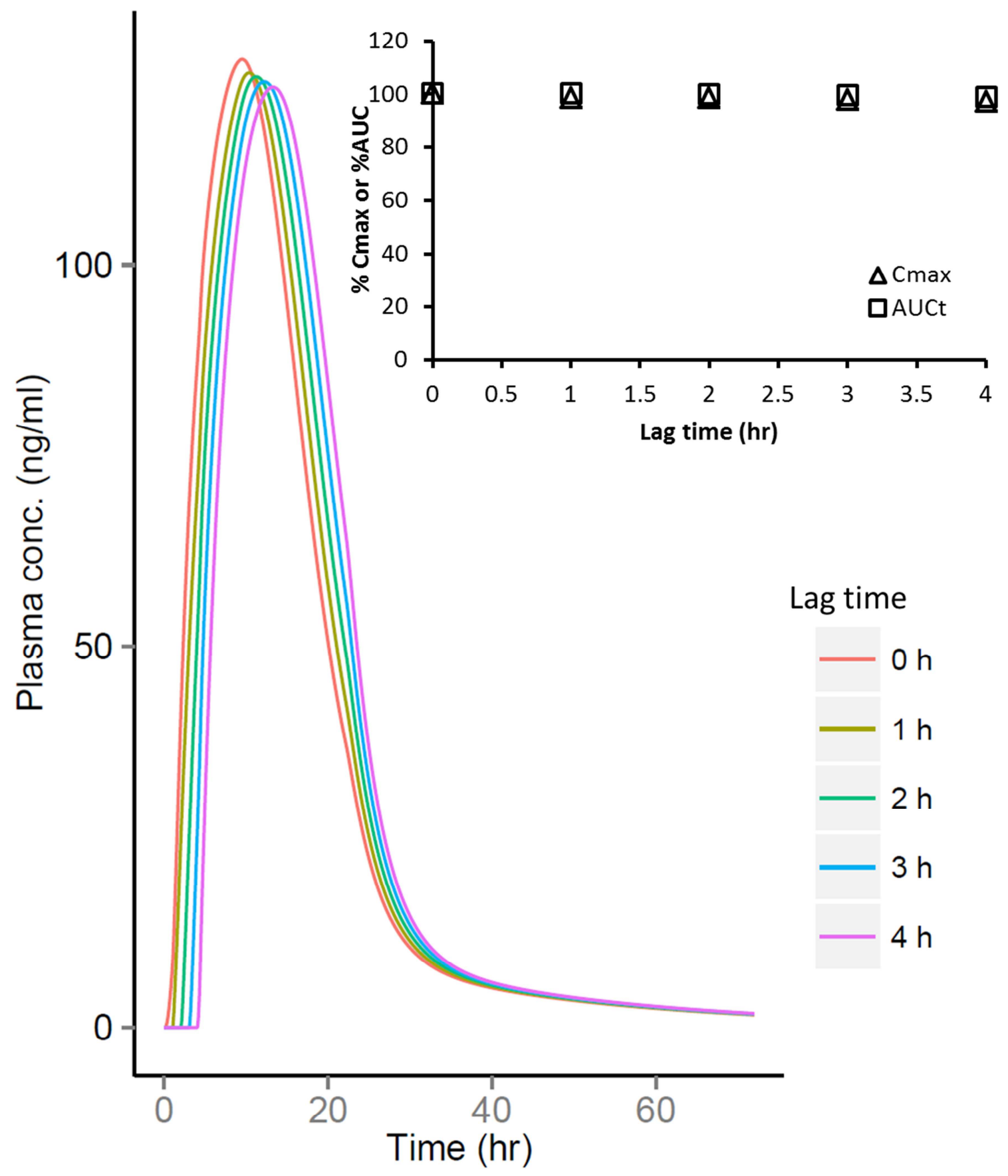

